\title{
MarineVerse Enables More People to Sail!
}

\author{
Michelle Mayumi Tizuka1, Greg Dziemidowicz² \\ 1Universidade Federal Fluminense - Brazil, ${ }^{2}$ Marine Verse - Australia \\ http://www.marineverse.com \\ mmtizuka@id.uff.br, greg@marineverse.com
}

\begin{abstract}
MarineVerse wants to promote and share the sport of sailing with the world by providing sailing education, growing as a sailor, socializing, and having entertainment using virtual reality technology. Current VR sailing applications are designed for Valve Index, HTC Vive, Oculus Rift, and Oculus Quest, and flagship products are MarineVerse Cup and VR Regatta.
\end{abstract}

Keywords: sailing; virtual reality; exergaming.

\section{Introduction}

MarineVerse (MV) started in January 2016. The idea was first tossed around in Hobart, Tasmania. The team had a chance to see the yachts finishing Sydney to Hobart race and relax in a peaceful apartment in North Hobart. After a brainstorming session, the project officially started.

Greg Dziemidowicz is the Lead Developer and grew up with sailing and technology. He started programming when he was eight years old and joined a sailing club when he was 12 . Over the years, many people have contributed to MarineVerse and its various projects like VR Regatta, Big Breezy Boat, and others. A full list of credits is available inside the press-kits of individual projects.

MarineVerse aims to promote and share the sport of sailing with the world. MV is doing it by providing sailing education and entertainment using virtual reality (VR) technology, building VR sailing applications for Valve Index, HTC Vive, Oculus Rift, and recently, Oculus Quest.

Also, as a vision, MarineVerse aims to promote sailing every day by providing engaging, interactive sailing experiences that motivate people to try the real sport. Besides, allowing senior sailors (Figure 1) to stay connected to the competition and their sailing friends by providing an accessible VR environment that offers ample social opportunities and facilitates rehabilitation. The use of VR technology in the areas of rehabilitation and therapy continues to grow, with encouraging results being reported for applications that address human physical, cognitive, and psychological functioning [1-4].

When everyone is using VR on a daily basis, MV also wants to be a safe harbor, where you can meet likeminded people, sail together, socialize and learn, growing as a sailor. One of the first researches involving sailing and virtual reality is reported on the work from Walls [5], where the ability of fourteen competitive helmsman of different skill levels to sail a standard course towards the wind (upwind) was assessed using a virtual reality sailing simulator. The results indicate that the test used can differentiate between variations in upwind sailing performance over a wide range of ability.

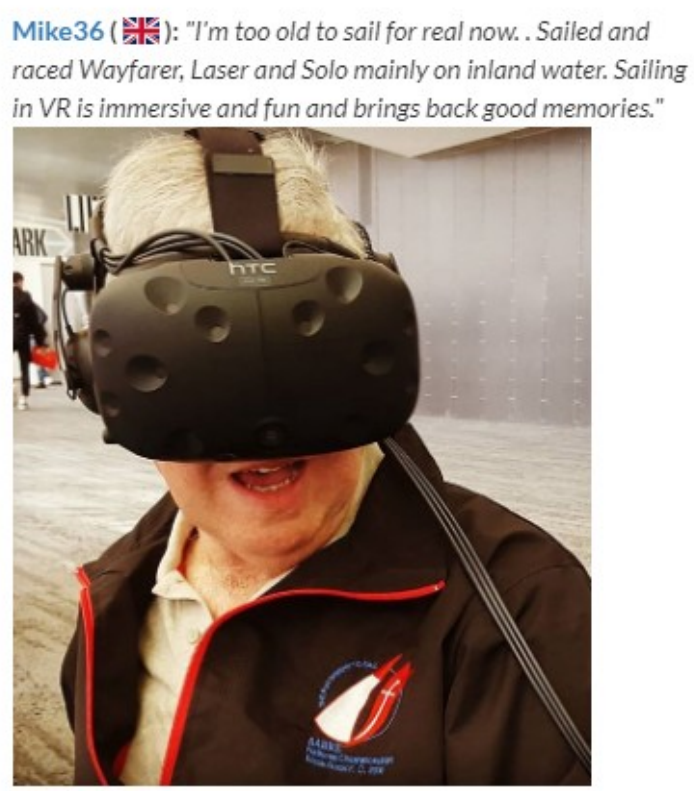

Figure 1. Screenshot from Marine Verse website. Allowing seniors to stay connected to the sport and their sailing friends.

\section{Main Projects}

MarineVerse flagship products are Marine Verse Cup [6] and VR Regatta - The Sailing Game [7] (Figure 2).

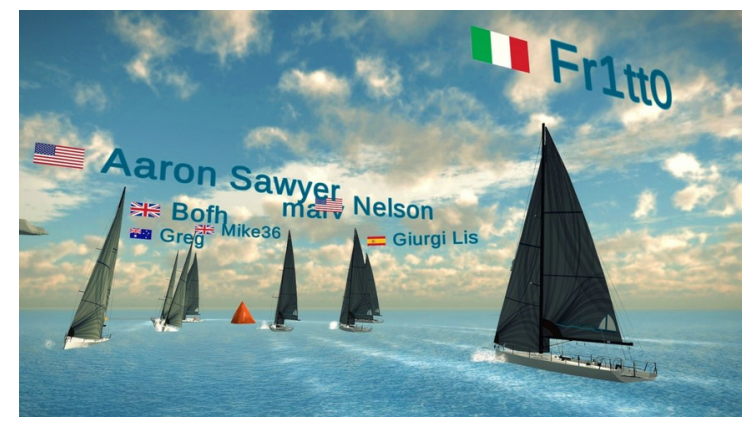

Figure 2. Screenshot at Yatch mode inside Marine Verse Cup Alpha.

VR Regatta was the debut project where it brings entertainment and education together through virtual reality. The idea is to create experiences to enable more people to sail through the creation of games, training 
experiences, and more. It is the leading VR sailing game for HTC Vive since 2016.

MarineVerse Cup is a competitive VR sailing game that brings multiplayer sailboat racing to every VR headset owner. Available to be joined daily and on weekly races (Figure 3), with varying wind conditions and courses that will test your sailing and racing skills.

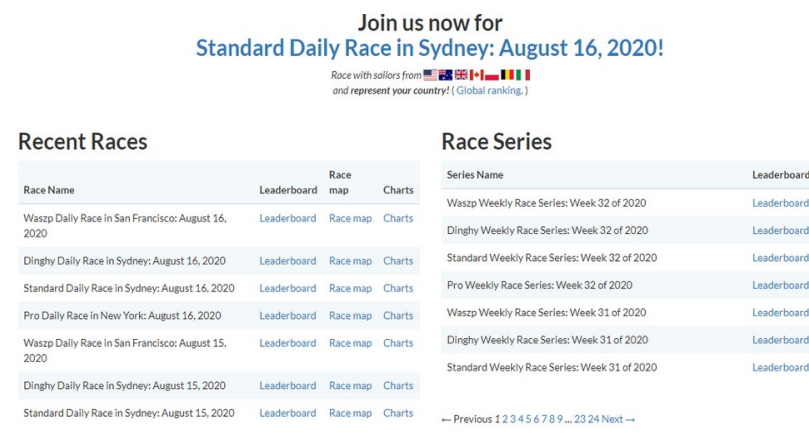

Figure 3. Screenshot at a racing board from MarineVerse Cup website.

Both simulation projects aim to promote engagement in the sporting spirit of sailing, encouraging exercise, a true exergame. Exergames are known as digital games combining training with gameplay, which can improve youths' health status and provide social and academic benefits [8] [9] [10].

However, it is perceived the potential of adults $50+$ and seniors also to improve health status, as another project from MV provides. Big Breezy Boat (B3) [11] is the first mobile project aiming to democratize sailing by bringing it to affordable virtual reality, and it complements the debut project - VR Regatta - The Sailing Game that brought sailing to high-end PC VR.

\section{Social and scientific contributions}

Virtual reality sailing enables MV to provide sailing training and education at your home, more cheaply, and quickly. The company considers it a fun and engaging tool to reinforce what you are already learning on the water. As a newbie, advantages are that you can go at your own pace, focusing on a given subject for as long as necessary. On top of that, an instructor will be able to join you in the virtual world, if you need anything specific explained.

In the case of an advanced sailor, all of the projects can connect you with experts from around the world while VR offers a unique learning environment. In the last five years, MV took place in seventeenth events to explore possible applications of virtual reality for promotion of the sport and potential future training applications, as recently as a partner in VR Fitness Summit 2020, presented by Virtual Athletics League (VAL).

As a social contribution, MarineVerse projects also have a high potential for social exergames to persuade seniors to increase physical activity [12]. The authors highlight that many elderly suffer from loneliness, making social interaction within exergames very important. In 2020, due to the pandemic situation, the number of adults $50+$ and seniors players at $\mathrm{MV}$ doubled during the lockdown period.

For instance, the experience of one of the virtual sailors' race, who started sailing in real life at eight years old and is now 54 and recently started playing on B3: "immediately after involved my 80 year old mother too on her own Go, living not far from each other but during COVID time, in $B B B$ relaxing, feeling like we used to feel when we were sailing, chatting and relaxing and being amazed!". When asked, "Who and why should try MarineVerse Cup?", the same sailor did not hesitate to say: "Just anyone who has the slightest thing with watersports/sailing because it is just very realistic amazing immersive competitive feeling, incl relaxing and escape into VR (...) The sailing, the behavior of the boat it is like in real life, you can learn how to sail here and or just continue or start a hobby that gets you involved for a lifetime.".

MV projects could eventually support research into these research areas, of how exergames can help to motivate the elderly to exercise more, focusing on possible social interactions in online exergaming and persuasive technologies. Enhancement of presence in a VR Sailing environment can also be conducted studies, as the work of [13] developed with a virtual training track with a large-scale visualization, rudder input, and haptic feedback on the mainsheet line.

Finally, MV also has plans to improve the Quality of Experience of participants using the system. Employing testing and using virtual questionnaires research for applied measurements that include not only game concepts such as flow, presence, video quality, and wellbeing but also sailing learning data analytics to attempt to identify success factors to guide designers of water exergaming systems.

\section{Partners \& Friends}

Marine Verse partners and supporters cannot fail to be mentioned [14]. MarkSetBot is the world's first robotic sailing mark. Led by a team of passionate sailors and innovators, MarkSetBot strives to grow the sport of sailing and improve the quality of race management through all that they do. MarkSetBots use GPS technology to hold position and zero in on a specific location, which may be dictated through an app or webpage. Changing courses becomes simple as marks are moved by dragging a waypoint and pressing a button. All this can be accomplished with precision greater than that of traditional marks.

MySail - Go Sailing is a crew finding and management platform for yacht racing. With MySail, skippers can easily organize and communicate with their regular race crew and find new sailors to join when they are short-handed. For sailors looking for their next - or first - crewing position, MySail provides a platform to connect with skippers and get out on the water more often. 


\section{References}

[1] Rizzo, A. S.; Kim, G. J. (2005) A SWOT analysis of the field of virtual reality rehabilitation and therapy. Presence: Teleoperators \& Virtual Environments 14(2): 119-146. DOI: $10.1162 / 1054746053967094$

[2] Howard, M. C. (2017) A meta-analysis and systematic literature review of virtual reality rehabilitation programs. Computers in Human Behavior 70: 317-327. DOI: 10.1016/j.chb.2017.01.013

[3] Schultheis, M. T.; Rizzo, A. A. (2001) The application of virtual reality technology in rehabilitation. Rehabilitation $\begin{array}{lll}\text { Psychology 46(3): } 296 . & \end{array}$ 10.1109/ATEE.2019.8724953

[4] Recio, A. C.; Becker, D.; Morgan, M.; Saunders, N. R.; Schramm, L. P.; McDonald III, J. W. (2013) Use of a virtual reality physical ride-on sailing simulator as a rehabilitation tool for recreational sports and community reintegration: a pilot study. American Journal of Physical Medicine \& Rehabilitation 92(12): 1104-1109. DOI: 10.1097/PHM.0000000000000012

[5] Walls, J., Bertrand, L., Gale, T., \& Saunders, N. (1998) Assessment of upwind dinghy sailing performance using a virtual reality dinghy sailing simulator. Journal of Science and Medicine in Sport 1(2): 61-72. DOI: 10.1016/S14402440(98)80014-5

[6] MarineVerse. VR - The Sailing Game. Available at https:// www.marineverse.com/vr-regatta-the-sailing-game
[7] MarineVerse. Marine Verse Cup. Available at https://www.marineverse.com/marineverse-cup

[8] Staiano, A. E.; Calvert, S. L. (2011) Exergames for physical education courses: Physical, social, and cognitive benefits. Child development perspectives 5(2): 93-98. DOI: $\underline{10.1111 / J .1750-8606.2011 .00162 . X}$

[9] Oh, Y.; Yang, S. (2010) Defining exergames \& exergaming. In: Proc. of Meaningful Play, 1-17.

[10] Sinclair, J., Hingston, P.; Masek, M. (2007) Considerations for the design of exergames. In: Proc. 5th Int. Conf. Computer Graphics and Interactive Techniques in Australia and Southeast Asia, p. 289-295.

[11] MarineVerse. Big Breezy Boat. Available at https://www.marineverse.com/big-breezy-boat

[12] Brox, E.; Luque, L. F.; Evertsen, G. J.; Hernández, J. E. G. (2011) Exergames for elderly: Social exergames to persuade seniors to increase physical activity. In: 2011 5th Int. Conf. Pervasive Computing Technologies for Healthcare (PervasiveHealth) and Workshops, p. 546-549. IEEE. DOI: 10.4108/icst.pervasivehealth.2011.246049

[13] Verlinden, J. C.; Mulder, F. A.; Vergeest, J. S.; de Jonge, A.; Krutiy, D.; Nagy, Z. et al. (2013) Enhancement of presence in a virtual sailing environment through localized wind simulation. Procedia Engineering 60: 435441. DOI: 10.1016/j.proeng.2013.07.050

[14] MarineVerse. Partners and Friends of MarineVerse. Available at https://www.marineverse.com/partners. 